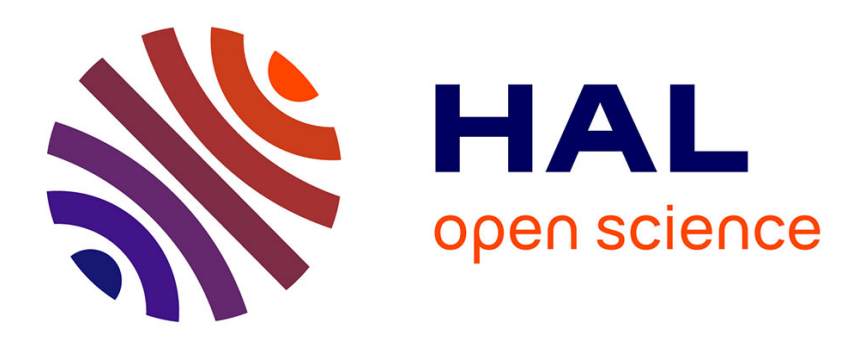

\title{
A comparison of reversible chemical reactions for solar thermochemical power generation
}

\author{
O.M. Williams
}

\section{To cite this version:}

O.M. Williams. A comparison of reversible chemical reactions for solar thermochemical power generation. Revue de Physique Appliquée, 1980, 15 (3), pp.453-461. 10.1051/rphysap:01980001503045300 . jpa-00244752

\section{HAL Id: jpa-00244752 https://hal.science/jpa-00244752}

Submitted on 1 Jan 1980

HAL is a multi-disciplinary open access archive for the deposit and dissemination of scientific research documents, whether they are published or not. The documents may come from teaching and research institutions in France or abroad, or from public or private research centers.
L'archive ouverte pluridisciplinaire HAL, est destinée au dépôt et à la diffusion de documents scientifiques de niveau recherche, publiés ou non, émanant des établissements d'enseignement et de recherche français ou étrangers, des laboratoires publics ou privés. 


\title{
A comparison of reversible chemical reactions for solar thermochemical power generation
}

\author{
O. M. Williams \\ Department of Engineering Physics, Research School of Physical Sciences, \\ The Australian National University, Canberra, Act, Australie \\ (Reçu le 7 septembre 1979, révisé le 10 décembre 1979, accepté le 12 décembre 1979)
}

\begin{abstract}
Résumé. - Les réactions chimiques réversibles qui se passent dans un système de transfert d'énergie thermochimique ont été proposés aux centrales solaires génératrices d'électricité pour résoudre non seulement le problème du transfert thermique entre le champ des capteurs solaires et la centrale, mais aussi potentiellement pour le stockage à long terme de l'énergie sans perte, par l'emmagasinage souterrain des produits de réaction. Plusieurs réactions ont été proposées pour générer la puissance électrique d'origine solaire thermochimique. Dans cette communication les critères de la thermodynamique et du génie chimique sont examinés pour comparer les réactions et ils sont appliqués aux quatre systèmes suivants fondés sur le mélange eau-méthane, le trioxide de soufre, l'ammoniac et le méthanol, chacun desquels est associé à une forte base industrielle. L'efficacité totale de la conversion énergie solaire thermique-énergie électrique est évaluée pour chaque système. Les processus composants du transfert thermique et du travail utile sont examinés pour montrer l'étendue du domaine dont il faut s'occuper pour calculer l'efficacité du système par rapport aux autres schémas de réaction qui soient possibles. Le système du trioxide de soufre offre l'efficacité la plus élevée $(23 \%)$ mais présente plusieurs ennuis pour la mise en œuvre. A défaut d'une comparaison détaillée des données qui optimisent les dépenses, on peut considérer que la réaction réversible d'ammoniac présente le meilleur compromis entre l'efficacité totale $(19 \%)$ et la difficulté liée du génie chimique de l'installation pour être un choix propice à la première génération des centrales solaires thermochimiques.
\end{abstract}

\begin{abstract}
Reversible chemical reactions operating in a thermochemical energy transfer system have been proposed for solar electricity generation in order to solve not only the problem of energy transport from the solar collection field to a central power plant, but also potentially the long term lossless energy storage problem through underground storage of the reaction products. A number of reactions have been proposed for solar thermochemical power generation and in this paper the thermodynamic and chemical engineering criteria for comparing the reactions are examined and are applied to the four prominent systems based on water-methane, sulphur trioxide, ammonia and methanol, each of which is associated with a broad industrial base. The overall efficiency for conversion from the solar thermal input to electricity is evaluated for each system and the component processes of heat transfer and work production are examined in order to highlight the areas that must be given special attention in calculating the system efficiency when alternative reaction schemes are considered. The sulphur trioxide system has the highest efficiency of $23 \%$ but is associated with several areas of concern regarding the practicalities of implementation and their effect on capital cost. In the absence of detailed comparative cost optimization data, it is considered that the system based on ammonia dissociation/synthesis has the best blend of overall efficiency $(19 \%)$ and moderate level of chemical engineering difficulty to be a good choice for first generation solar thermochemical power generation.
\end{abstract}

1. Introduction. - One of the more novel approaches to the problem of transporting energy from a solar collection field to a central plant in a form suitable for large scale electricity generation is the use of reversible chemical reactions in a solar thermochemical energy transfer system. In such a system, as outlined in figure 1 , concentrated solar radiation is converted into high grade heat at a single focal absorber as in the central solar receiver system, or at the multiple absorbers of a distributed paraboloidal collector array. The high grade heat is in turn converted into chemical energy in promoting an endothermic reaction and the reaction products are transported through pipes to a central plant. A counterflow heat exchanger connects each high temperature absorber to the pipe system, thus ensuring that both the reac- 


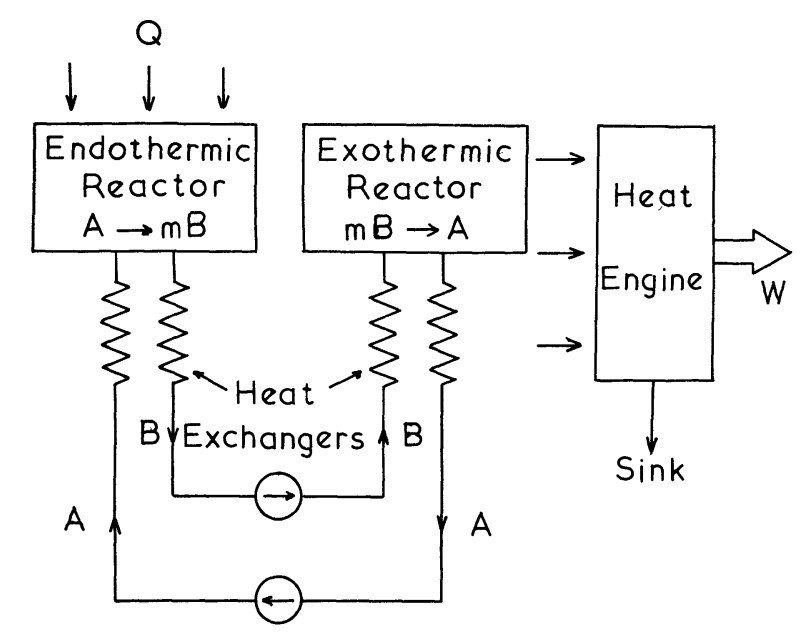

Fig. 1. - Basic outline of a thermochemical energy transfer system (without phase separation).

tant and product working fluids are pumped between the collector field and the central plant at essentially ambient temperatures. High grade reaction heat is regenerated at a central exothermic reactor during the reverse procedure and is converted into electricity through a standard steam-driven turbo-alternator. There is potential also for long term lossless energy storage through storage of the reaction products from the endothermic reaction.

A large number of reversible chemical reactions have been proposed for thermochemical energy transfer and storage [1], but few are suitable for operation in a first generation solar thermochemical power plant. The reaction schemes considered in this paper are those for which there is widespread industrial working knowledge available ; namely, steam reforming of methane [2-5] and dissociation of sulphur trioxide [6-8], ammonia [9-13] and methanol [14, 15]. The alternative system based on carbon dioxide reforming of methane is regarded here as being described as part of the wider stoichiometry of the same system as steam reforming of methane. No system involving solids with the inherent difficulties of transport of reactants is considered in this study.

Selection of the optimum reaction system for solar thermochemical power generation is governed by both the overall thermodynamic performance and the chemical engineering criteria which determine practicality and cost of operation. In a thorough design analysis, these factors are combined in terms of the overall cost-effectiveness which is normally expressed as an installed capital cost or as the busbar cost of the electricity produced. At the present stage of development, there is insufficient comparative data for such a complete analysis. Nevertheless, it is important that an early guide as to the viability of each system be obtained. In this paper, the thermodynamic and chemical engineering criteria are examined separately in order to provide an early comparison among the four systems listed above. In addition, the study provides guidance as to the important features which must be examined carefully when new candidate reaction systems are proposed. In particular, the methods available for preliminary thermodynamic screening of candidate reactions are assessed, and the mechanisms of heat transfer and work production which serve to define the overall system efficiency are examined in detail.

The efficiency for conversion from the high grade solar thermal input to electricity generated at a central plant is calculated as $15 \%, 23 \%, 19 \%$ and $16 \%$ for the water-methane, sulphur trioxide, ammonia and methanol systems respectively. It is assumed that a modern multistage steam turbine is available for work production. While the sulphur trioxide system has the highest efficiency, it is not necessarily the optimum choice for a first generation plant, principally because of the practical difficulties associated with the corrosive nature of sulphur trioxide at the elevated source temperatures and its potential for solidification in the fluid reticulation network. The water-methane system is suitable for industrial heat distribution from a central power plant where use may be made of the capacity for cogeneration of electricity and medium grade condensation heat, but is less suitable for solar power generation where there is less scope for utilization of the condensation heat. In the absence of detailed cost optimization data, the system based on ammonia appears to have the best blend of overall efficiency and moderate level of engineering difficulty to be a good choice for a first generation solar thermochemical power plant. The methanol system is regarded as being inferior on the twin bases of lower efficiency and greater chemical engineering difficulty caused by side-product formation.

The thermodynamic assessment of reversible chemical reactions for solar thermochemical power generation is discussed in section 2 and in section 3 the system efficiencies for the four candidate reactions are evaluated, with particular emphasis on identification of the sources of irreversibility and energy loss occurring in the individual processes of heat transfer and work production. The chemical engineering aspects of the four candidate systems are outlined in section 4 in order to provide sufficient information for an early comparison among the systems and a benchmark to which new systems proposed in the future may be compared.

2. Thermodynamic comparison of systems. - A reversible reaction system suitable for first generation solar thermochemical power generation using steam turbines for work recovery should absorb solar thermal energy at temperatures lower than $1000^{\circ} \mathrm{C}$ in order to lessen the problems of materials limitation and absorber losses, and should further be capable of delivering heat to the turbine inlet at typically $500^{\circ} \mathrm{C}$. In this section, the thermodynamic criteria for preli- 
minary screening of candidate reactions are assessed, and the available thermodynamic equilibrium data are examined in more detail for the four systems of interest in this study, in relation to their respective capabilities of achieving the desired operation within the specified temperature range.

2.1 Preliminary SCREENING OF REACTIONS. Two parameters are useful for initial screening of reversible reactions for solar thermochemical power generation. The first is the turning temperature $T^{*}$ $[1,16]$ which is defined as the temperature at which neither the forward nor the reverse reaction is thermodynamically favoured. The form of $T^{*}$ is derived simply by applying the definition of Gibbs' free energy to the condition that at $T^{*}$

$$
\Delta G\left(T^{*}, P\right)=0
$$

where $\Delta G$ in the Gibbs' free energy change for the reaction, in which case

$$
T^{*}=\Delta H\left(T^{*}, P\right) / \Delta S\left(T^{*}, P\right) .
$$

Here, the numerator and denominator represent respectively the reaction enthalpy and entropy at pressure $P$ and at $T^{*}$. The second screening parameter is the maximum work recovery efficiency $\eta_{\mathrm{R}}^{\max }$ for the exothermic reaction, which is obtained from standard chemical thermodynamics as

$$
\eta_{\mathrm{R}}^{\max }=\Delta G\left(T_{\mathrm{S}}, P\right) / \Delta H\left(T_{\mathrm{S}}, P\right)
$$

since $\Delta G\left(T_{\mathrm{S}}, P\right)$ is the maximum available work. $T_{\mathrm{S}}$ represents the ambient or sink temperature and attainment of the maximum work implies that not only must the exothermic heat exchanger operation be reversible, but that the exothermic reaction must also proceed reversibly. That is, the reaction must proceed so that chemical equilibrium is maintained at all temperatures, the reaction temperature falling as the reaction progresses such that it always equals the temperature defined by the equilibrium mixture. The reaction then follows the dashed equilibrium line shown in figure $4 a$ below. All the reaction heat and all heat imbalances in the heat exchanger region must be converted into maximum work by use of a system of Carnot engines available throughout the temperature range.
The significance of both $T^{*}$ and $\eta_{\mathrm{R}}^{\max }$ is that they may be estimated to a satisfactory approximation for a given reaction by use of standard reference data alone [16]. Values for the four reactions of interest in this paper are listed in table I. Stoichiometric proportions have been assumed for both the reactant and product mixtures, except for the calculation of $\eta_{\mathbb{R}}^{\max }$ for the water-methane system. In both steam reforming of methane and methanation it is common practice to use excess water to prevent carbon deposition on the catalyst and, in the steam reforming case, to drive the equilibrium in favour of methane consumption. In this case, the maximum available work has been evaluated from the energy histograms tabulated in [5] as

$$
\Delta G\left(T_{\mathrm{S}}, P\right)=\int_{T_{\mathrm{S}}}^{\infty}\left(1-\frac{T_{\mathrm{S}}}{T}\right) d[\Delta H(T, P)]
$$

for a methanator operating at equilibrium with $50 \%$ excess water in the feedstock. The information available from the histograms is essentially the same as that shown below for the ammonia system in figure $4 a$.

The parameters of table I reveal the contrast between the water-methane and sulphur trioxide systems on the one hand where $500^{\circ} \mathrm{C}$ heat may be delivered without difficulty and, on the other hand, the ammonia and methanol systems which are characterised by lower values of $T^{*}$ and $\eta_{\mathrm{R}}^{\max }$. These latter are the better suited for solar absorber operation at moderate temperatures where energy losses are small and materials limitations are less severe. A further point of interest is that the values of $\eta_{\mathbb{R}}^{\max }$ for water-methane and sulphur trioxide exceed the Carnot efficiency of 0.64 equivalent to $550^{\circ} \mathrm{C}$ heat delivery which is the maximum working temperature in modern steam turbine practice. For these systems, therefore, heat would necessarily be degraded to suit the turbine inlet requirements and the apparent disparity in work availability is then reduced among the four systems of table I.

Although calculation of $T^{*}$ or $\eta_{\mathrm{R}}^{\max }$ is valuable for initial screning of proposed candidate reactions for solar thermochemical energy transfer, particularly in view of the ease of calculation using the minimum amount of reference data, a note of caution must be sounded when more detailed comparisons are requir-

\begin{tabular}{|c|c|c|c|c|c|}
\hline Reaction & $\begin{array}{c}P \\
\mathrm{MPa}\end{array}$ & $\begin{array}{c}\Delta H_{298}^{0} \\
\mathrm{~kJ} \mathrm{~mole}^{-1}\end{array}$ & $\underset{\mathrm{J} \mathrm{mole}^{-1} \mathrm{~K}^{-1}}{\Delta G_{298}}$ & $\begin{array}{l}T^{*} \\
\mathrm{~K}\end{array}$ & $\eta_{\mathbf{R}}^{\max }$ \\
\hline - & - & - & - & - & - \\
\hline$\frac{1}{2} \mathrm{H}_{2} \mathrm{O}+\frac{1}{2} \mathrm{CH}_{4} \rightleftharpoons \frac{3}{2} \mathrm{H}_{2}+\frac{1}{2} \mathrm{CO}$ & 4.0 & 103.1 & 107.3 & 1285 & 0.71 \\
\hline $\mathrm{SO}_{3} \rightleftharpoons \mathrm{SO}_{2}+\frac{1}{2} \mathrm{O}_{2}$ & 0.3 & 98.2 & 128.7 & 1000 & 0.70 \\
\hline $\mathrm{NH}_{3} \rightleftharpoons \frac{3}{2} \mathrm{H}_{2}+\frac{1}{2} \mathrm{~N}_{2}$ & 30.0 & 45.9 & 99.2 & 751 & 0.54 \\
\hline $\mathrm{CH}_{3} \mathrm{OH} \rightleftharpoons 2 \mathrm{H}_{2}+\mathrm{CO}$ & 30.0 & 90.7 & 222.6 & 631 & 0.52 \\
\hline
\end{tabular}
ed. There is no information available from either $T^{*}$

Table I. - Preferred reaction pressure and thermochemical energy transfer screening data calculated for the four candidate reactions for solar thermochemical power generation. Sink temperature $T_{\mathrm{S}}=300 \mathrm{~K}$. 
or $\eta_{\mathrm{R}}^{\max }$ to define the rapidity of the change from exothermic to endothermic behaviour and the calculations assume stoichiometric mixtures and essentially complete reaction without phase separation, both of which can have an important bearing on the suitability of particular reaction for thermochemical energy transfer. In order to make a more detailed comparison it is necessary to examine the full equilibrium data available for each system, as discussed in the following paragraph.

2.2 ChemiCAl EQUILIBRium DATA. - The chemical equilibrium data [17-20] for each of the four reactions of interest are shown in figure 2 where the ordinate represents the product gas mole fraction in the dry equilibrium mixture (excluding water content), with product gas defined by the right hand side of the reactions listed in table $\mathrm{I}$. The effect of the accompanying water-gas shift reaction has been taken into account for calculation of the water-methane curve.

It is seen that there is a good correspondence between the midpoints of the curves and the values of $T^{*}$ listed in table I, except for the water methane system where the non-stoichiometric mixing of reactants has had a significant effect in displacing the equilibrium to lower temperatures, to the extent that the system is better suited for solar thermochemical absorber operation than that based on sulphur trioxide. As discussed above, both systems are wellsuited to $500{ }^{\circ} \mathrm{C}$ heat recovery. In contrast, the methanol equilibrium curve covers the lowest temperature range and is not well-suited to $500{ }^{\circ} \mathrm{C}$ heat recovery. The ammonia curve is similarly displaced towards low temperatures and would also be regarded as unsuitable for $500{ }^{\circ} \mathrm{C}$ heat recovery were it not for incorporation of a gravitational settling tank in the

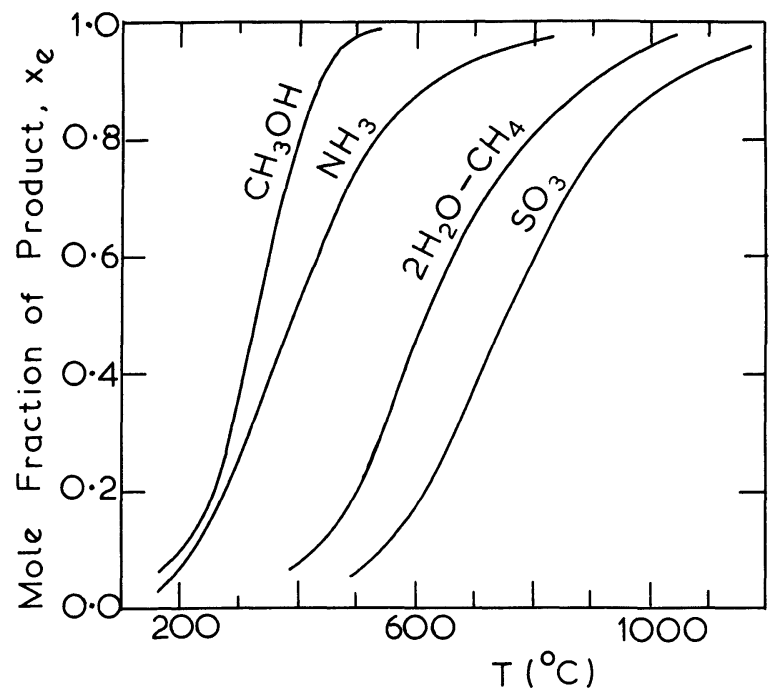

Fig. 2. - Temperature variation of the equilibrium compositions for the reversible reactions based on methanol (30 MPa), ammonia $(30 \mathrm{MPa})$, water-methane (4.0 MPa), and sulphur trioxide $(0.3 \mathrm{MPa})$. The ordinate represents the product gas mole fraction in the dry equilibrium mixture. thermochemical system, as shown in figure 3. This feature ensures that both the endothermic and exothermic reactors are fed with relatively pure reactant and product mixture respectively, irrespective of the extent to which the reactions occur. In particular, the ammonia synthesis reaction can proceed at $500^{\circ} \mathrm{C}$ and $25 \%$ conversion, as typical of ammonia manufacturing plant. The dissociation reaction proceeds close to completion at $750^{\circ} \mathrm{C}$ [21].

Of the four systems, therefore, only the methanol system is unsuitable for $500{ }^{\circ} \mathrm{C}$ heat delivery, and of the remaining three systems, that based on ammonia has the lowest temperature for solar thermochemical absorber operation. The absorbers for both the watermethane and sulphur trioxide systems would need to operate in the $900-950{ }^{\circ} \mathrm{C}$ region in order to achieve high endothermic reaction conversions. This temperature range is higher than ideally desirable, but is not excessively high in the sense that effective solar thermochemical absorber operation is impossible. Only the methanol system is therefore regarded as doubtful for solar thermochemical power generation on the basis of the equilibrium data assessment.

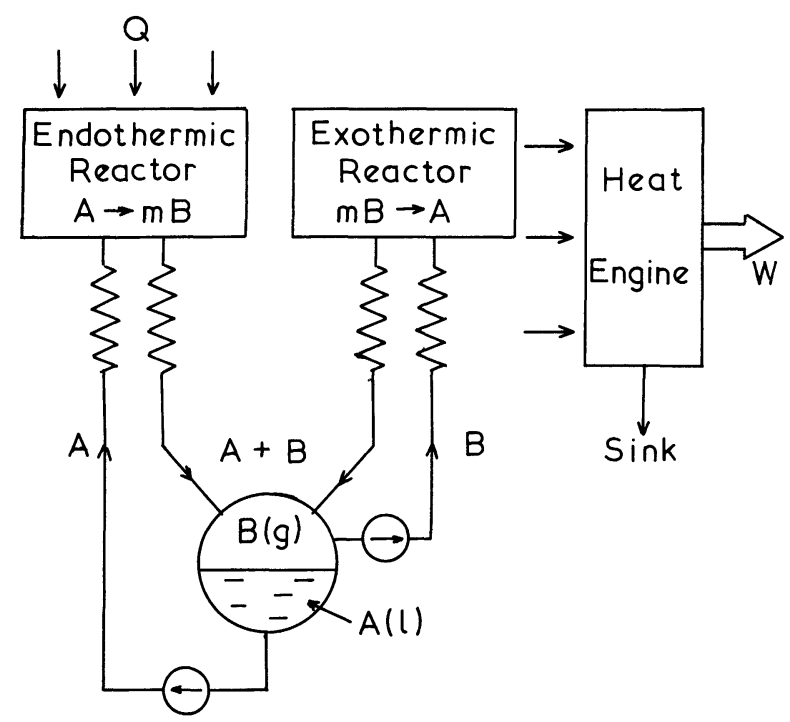

Fig. 3. - Basic outline of a thermochemical energy transfer system with phase separation of liquid reactant $\mathbf{A}$ and gaseous product $\mathbf{B}$.

3. System efficiency calculation. - The overall efficiency for network production from the solar thermal input is defined as

$$
\eta_{0}=W / Q=\eta_{\mathrm{a}} \eta_{\mathrm{st}} \eta_{\mathrm{hr}} \eta_{\mathrm{e}} \eta_{\mathrm{ext}}
$$

where the factors represent respectively the solar absorber efficiency $\eta_{\mathrm{a}}$, the chemical energy storage efficiency $\eta_{\mathrm{st}}$, the high grade heat recovery efficiency $\eta_{\mathrm{hr}}$ at the central plant, the practical heat engine efficiency $\eta_{\mathrm{e}}$, and the external work availability factor $\eta_{\text {ext }}$ which accounts for the internal consumption of work by fluid circulation pumps and solar collector actuators. In the following sections, the efficiency 
factors in (5) are evaluated for the four thermochemical systems of table I and the overall thermodynamic efficiencies are compared.

3.1 SOlAR ABSORBER EFFICIENCY. - The efficiency of an ammonia-based solar thermochemical cavity absorber located at the focus of a $10 \mathrm{~m}^{2}$ paraboloidal collector has been calculated recently by Williams [21] and the estimates therein serve as a basis for comparison among the four thermochemical energy transfer systems. Efficiency factors of 0.99 are assumed for the radiation capture ratio by the cavity aperture and for the cavity absorbtivity. Conduction, convection and reradiation losses amount respectively to $1.7 \%$, $0.4 \%$ and $3.3 \%$ of the radiation input to an ammoniabased absorber operated at $750{ }^{\circ} \mathrm{C}$ at the focus of a paraboloidal dish characterised by an area concentration ratio of 2000 . Shading of the dish by the focal absorber amounts to $0.6 \%$ of the incident radiation and when all effects are included, an overall absorber efficiency of $92 \%$ is obtained.

The absorbers for both sulphur trioxide and the water-methane reactions would be expected to operate at a wall temperature of typically $950^{\circ} \mathrm{C}$ where reradiation losses would be approximately doubled. Reradiation losses can of course be reduced by incorporating modifications such as a Francia window, but only at the expense of increased capital cost. Such refinements are not considered here in order to retain a common basis for comparison. The increased operating temperature leads also to increased conduction and convection losses and furthermore, for the sulphur trioxide system where the reactor and heat exchanger are liable to be of larger size in order to avoid significant pressure drop in the fluid stream, both the conduction and shading losses are expected to be further increased. These factors have been included in the assessment of solar absorber efficiencies listed below in table II.

Table II. - Thermochemical energy transfer efficiency factors comprising the overall efficiency $\eta_{0}$ for solar thermal to electricity conversion. The efficiency for insolation to electrical conversion is obtained by multiplying $\eta_{0}$ by the reflectivity and cosine factor appropriate to the solar concentrator.

\begin{tabular}{lcccccc}
\multicolumn{1}{c}{ Reaction } & $\eta_{\mathrm{a}}$ & $\eta_{\mathrm{st}}$ & $\eta_{\mathrm{hr}}$ & $\eta_{\mathrm{e}}$ & $\eta_{\text {ext }}$ & $\eta_{\mathrm{o}}$ \\
$\mathrm{H}_{2} \mathrm{O}-\mathrm{CH}_{4}$ & 0.88 & 0.62 & 0.90 & 0.35 & 0.93 & 0.16 \\
$\mathrm{SO}_{3}$ & 0.86 & 0.90 & 0.90 & 0.35 & 0.93 & 0.23 \\
$\mathrm{NH}_{3}$ & 0.92 & 0.99 & 0.68 & 0.33 & 0.93 & 0.19 \\
$\mathrm{CH}_{3} \mathrm{OH}$ & 0.92 & 0.95 & 0.70 & 0.26 & 0.93 & 0.15
\end{tabular}

3.2 Heat EXChanger Balances. - The counterflow heat exchangers incorporated in both the endothermic and exothermic reaction plants are critical components in ensuring that a satisfactory overall system efficiency is attained and it is important that their operation be examined carefully, particularly for the liquid/gas systems where significant thermal mismatches occur. The exchangers have the dual role of providing feed stream preheat and simultaneous product stream cooling and are essential for minimising thermodynamic irreversibility.

Complete heat transfer reversibility implies that heat released at each temperature from the return stream must be absorbed by the feed stream at the same temperature or alternatively used elsewhere in the thermochemical system without temperature degradation. Practical heat exchangers are nonideal in the sense that spontaneous heat transfer must occur across a finite temperature difference. However, this source of irreversibility is often dominated by the irreversibilities that can occur because of differing specific heats of the feed and return fluids. The heat imbalances occurring in a liquid/gas thermochemical energy transfer system are illustrated in figure 4 by the temperature-enthalpy diagram for the ammonia system at $30 \mathrm{MPa}$ in which both the endothermic and exothermic reaction loops are shown together with the corresponding heat exchanger temperature profiles.

3.2.1 Endothermic reaction loop. - The major irreversibility occurring in the endothermic loop shown in figure $4 a$ occurs through the absorption of the latent heat of vaporisation of the ammonia feed and the release at lower temperatures of the latent heat of condensation of the residual ammonia in the return stream. The temperature difference between the vaporisation and condensation heats is related closely to the requirement that the work of phase separation must be developed internally within the system, as discussed elsewhere $[12,13]$. The corresponding heat exchanger temperature profile in figure $4 b$ is characterised by a steep increase in the high temperature zone where the return fluid stream is unable to supply the latent heat requirements of the ammonia feed, and by the converse curvature at low temperatures where ammonia condensation occurs in the return stream. A finite amount of low grade heat is carried from the heat exchanger by the exiting fluid stream. In a solar power plant it would be unlikely that this heat could be used economically and instead it would most probably be wasted to the surroundings.

In order to quantify the energy wastage, the energy storage efficiency has been defined elsewhere [11] as

$$
\eta_{\mathrm{st}}=\frac{\text { Stored enthalpy }}{\text { Net thermal input from source }}
$$

and has been calculated for the ammonia dissociation reaction [12]. It is found that $\eta_{\mathrm{st}}$ exceeds $90 \%$ provided that the endothermic reaction extent exceeds $60 \%$ ( $60 \%$ by weight of $3: 1$ hydrogen-nitrogen mixture) and equals unity for reaction extents exceeding $85 \%$, corresponding to the case where the partial pressure of ammonia in the return stream is less than the 

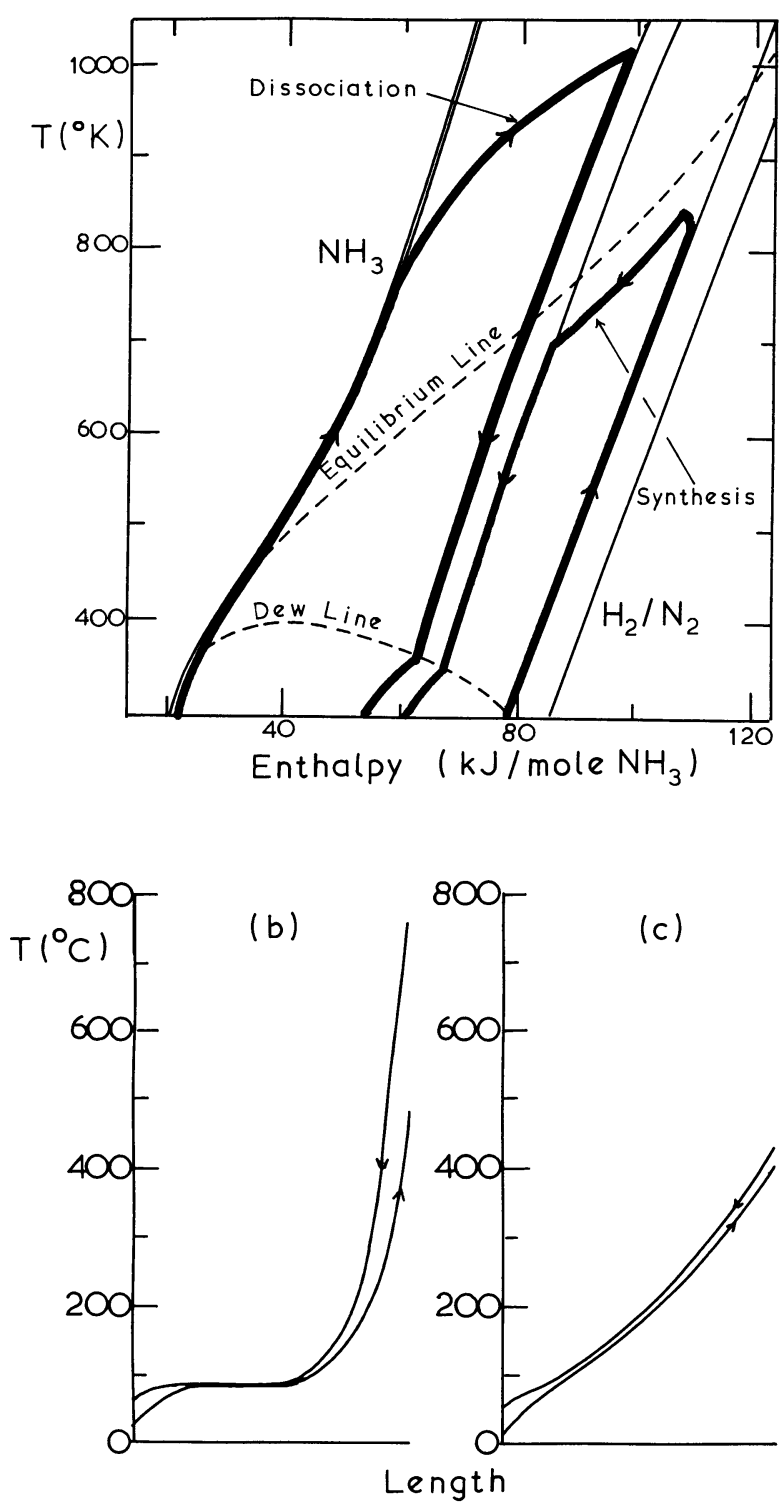

Fig. 4. - a) Temperature-enthalpy diagram for the thermochemical energy transfer system based on ammonia dissociation/synthesis at $30 \mathrm{MPa} . b$ ) Temperature profile for the dissociation heat exchanger. c) Temperature profile for the synthesis heat exchanger.

saturation vapour pressure of ammonia at ambient temperatures; that is, where no phase separation occurs in the return stream. Thus, provided that the flow rate to each absorber is adjusted so that the dissociation reaction extent exceeds $85 \%$, the energy wastage from the ammonia dissociation loop due to phase separation can be reduced to zero.

The same effect occurs in the water-methane system but in this case, condensation in the return stream is unavoidable because of the need to deliver the steam reforming reactor with excess water in order to drive the reaction towards complete methane consumption and at the same time to prevent carbon deposition on the catalyst. In this case, water is evaporated from the feed stream within a temperature range consistent with a high water vapour partial pressure and recon- denses within a lower temperature range consistent with the lower partial pressure in the return stream. Since the water is never fully consumed, the energy storage efficiency necessarily assumes a value lower than unity.

The problems of energy imbalance in the heat exchangers has also been recognised as important for the water-methane system by the investigators of the General Electric Company [5] who have prepared energy histograms which may readily be interpreted in determining values of energy storage efficiency. A value of $\eta_{\mathrm{st}}$ equal to 0.62 corresponding to heat rejection at typically $150^{\circ} \mathrm{C}$ is derived from the energy histograms for a $3: 1$ water-methane feedstock, showing that the problem of energy imbalance in the endothermic reaction loop is particularly severe. Indeed, the seriousness of the problem is sufficient to cast considerable doubt on the promise of the water-methane system for first generation solar thermochemical power generation. The same problem occurs in the alternative industrial heat and electricity distribution application for which the system is primarily being developed but there is considerably more scope there for utilizing the medium grade condensation heat.

The values for sulphur trioxide and ammonia have been calculated from the effect of heat exchanger nonideality. The overall heat transfer coefficient is typically 100 times smaller for sulphur trioxide than for ammonia because of the lower pressure operation and the need to avoid significant frictional pressure drop leads to the requirement for rather larger gas flow channels. Accordingly, the heat exchanger area in the system proposed by Chubb [6] is 100 times larger than the area required to exchange the same power for the ammonia system [21]. The values of $\eta_{\text {st }}$ in table II reflect the lower area differential of a factor of ten which is likely to give a more sensible cost comparison, given that the ceramics used for sulphur trioxide heat exchanger construction may ultimately prove less expensive than the stainless steel required for high pressure ammonia. The methanol value in table II is dominated by low grade heat rejection which is difficult to avoid because of the low saturation vapour pressure of methanol at ambient temperatures.

3.2.2 Exothermic reaction loop. - A similar condensation effect occurs in the exothermic reaction loop, as seen by reference to figures $4 a$ and $4 c$. It is convenient to define the high grade heat recovery efficiency

$$
\eta_{\mathrm{hr}}=\frac{\text { High grade heat recovered }}{\text { Stored enthalpy }} .
$$

For the ammonia system, $24 \%$ of the stored enthalpy is delivered as low grade heat of condensation, a result again of the requirement that work of separation must be developed within the system, as discussed elsewhere [13]. An additional $10 \%$ loss of high grade 
heat occurs because of heat exchanger nonideality, corresponding to a mean temperature difference of $20^{\circ} \mathrm{C}$ in the single phase region. This loss is significantly higher than for the dissociation heat exchanger because of the comparatively low ammonia conversion of typically $25 \%$.

The value of $\eta_{\mathrm{hr}}$ for the sulphur trioxide system corresponds to a temperature difference of $100{ }^{\circ} \mathrm{C}$ at the cold end of the exchanger, the magnitude again reflecting the low heat transfer coefficient that can be achieved in a cost-effective exchanger. For watermethane, the value has been calculated from the energy histograms of [5] with the heat of condensation rejected at typically $200{ }^{\circ} \mathrm{C}$ being rated of an equivalent work basis at half the work value of the high grade heat of reaction. This criterion has been adopted since Organic Rankine Cycle engines are available for converting $200{ }^{\circ} \mathrm{C}$ process heat into electricity at efficiencies in the range $15-20 \%$.

3.3 HeAT ENGINE EFFICIENCY. - It is clear from figure 2 that the exothermic reactions for both the water-methane and sulphur trioxide systems are capable of delivering heat at $500-550^{\circ} \mathrm{C}$ to the inlet of a steam turbine. We assume that a practical engine efficiency of 0.35 is achievable through use of a multistage steam turbine with reheat and regeneration.

It is also possible to deliver heat at $500^{\circ} \mathrm{C}$ from the ammonia synthesis reaction by tailoring the exothermic reactor profile to follow below the equilibrium line, as shown in figure $4 a$. The heat generated along the reactor length is transferred by counterflow heat exchanger to the boiler-superheater and reheat streams feeding the turbine inlets. Thus, an engine efficiency of approximately 0.33 can be achieved from the ammonia synthesis reaction heat. Heat from the methanol system would most likely be delivered at about $350^{\circ} \mathrm{C}$, corresponding to a reduced heat engine efficiency of typically 0.26 .

3.4 INTERNAL WORK REQUIREMENTS. - Part of the electricity generated is consumed within the power plant for driving the collectors and for pumping the working fluids around the endothermic and exothermic reaction loops. Pumping power required to overcome pipe friction in the fluid reticulation network of a $10 \mathrm{MWe}$ distributed power plant has been shown by Williams [22] to consume less than $1 \%$ of the output power. Further power is required in the field to overcome the pressure drop across each absorber/heat exchanger and to drive each collector. In the absence of detailed information we adopt the conservative estimate that these effects consume $2 \%$ of the power production.

Power consumption in the exothermic loop is expected to be consistent with that associated with commercial process plant. Based on an ammonia production plant of 1000 tonnes/day capacity (equivalent to $10 \mathrm{MWe}$ electricity production) and a pressure drop of typically $1 \mathrm{MPa}$ across the synthesiser loop, circulation pump power consumption amounts to approximately $0.5 \mathrm{MWe}$, or $5 \%$ of the anticipated electrical output. This figure is adopted as representative of the central plant power requirements for the four candidate systems under present study.

3.5 OVERALl SYSTEM EFFICIENCY. - The efficiencies calculated in the above sections are compared in table II and an overall system efficiency is calculated according to (5). The overall system efficiencies must be multiplied by the collector reflectivity and appropriate cosine factor in order to obtain the efficiency for solar to electrical conversion.

The data of table II show that the four reaction systems which have been considered here for first generation solar thermochemical power production all give system efficiencies in excess of $15 \%$. The data also highlights those areas of energy transfer and work production that need special attention for a given system. For example, the question arises as to whether or not it is valuable to recover the $150{ }^{\circ} \mathrm{C}$ heat released by each endothermic heat exchanger in the water-methane system, an effect that so drastically affects the value of energy storage efficiency and hence overall system efficiency. The lower heat recovery temperature of the methanol system is also highlighted through the low value of engine efficiency and in this case, one is lead to the conclusion that the methanol system is inferior to the similar system based on ammonia.

Of the three remaining systems, that based on sulphur trioxide has the highest overall efficiency of $23 \%$ with the ammonia system second at $19 \%$. The differences in efficiency are not sufficient to make a definitive choice of optimum system, given that there are also significant differences of a chemical engineering nature. The ultimate criterion for selection must be cost effectiveness and this can only be assessed by thorough design optimizations which take into account not only the system efficiency but also the practicality and cost of achieving the desired operation and design specifications. The latter effects are considered in the following section.

4. Chemical engineering data. - Although it is not within the scope of the present work to provide a detailed chemical engineering assessment of each of the four candidate reaction systems, it is of value to identify those design characteristics that have an important bearing on the practicality of operation. In this section, the design characteristics are listed for the four reaction systems of interest.

4.1 WATER-METHANE. - Steam reforming of methane occurs inevitably in conjunction with the water gas shift reaction [17]

$$
\mathrm{CO}+\mathrm{H}_{2} \mathrm{O} \rightleftharpoons \mathrm{CO}_{2}+\mathrm{H}_{2}
$$

for which excess water must be supplied. The excess water is necessary also to prevent carbon deposition on the catalyst. 
- Nickel alloys are required for construction of the steam reforming solar absorbers operated at typically $950^{\circ} \mathrm{C}$.

- Variations in isolation lead to significant changes in the steam reforming product mixture unless the flow to each solar absorber is servoed to the isolation level.

- The system is well-suited to $500^{\circ} \mathrm{C}$ heat delivery and to cogeneration of electricity and $200{ }^{\circ} \mathrm{C}$ process heat.

- Although the toxicity of carbon monoxide is high, it is easily dispersed. There is considerable industrial experience related to process plant applications.

- Steam reforming and methanation catalysts (usually nickel on a support such as alumina) are readily available.

- Energy storage can be implemented by underground storage of the gas mixtures, although carbon dioxide would need to be removed and separate storage provided for the methane-rich and hydrogenrich mixtures.

4.2 SUlPHUR TRIOXIDE. - No thermodynamically stable side-products are formed.

- The corrosive nature of sulphur trioxide at high temperatures $\left(950^{\circ} \mathrm{C}\right)$ leads to the necessity for inert ceramic materials to be used for solar absorber construction. Further research is required to ascertain whether a long-life economically viable solar absorber can be developed.

- Poor heat transfer because of the low pressure operation leads to high heat exchanger costs.

- The feed rate to each solar absorber needs to be servoed to the input isolation in order to maintain output mixture integrity.

- The feed and return pipelines must be lagged and maintained above $90^{\circ} \mathrm{C}$ in order to prevent sulphur trioxide condensation and potential solidification. There are startup problems if overnight solidification is allowed and alternatively, increased capital costs and internal energy consumption if the sulphur trioxide is kept hot overnight.

- The system is well-suited to $500^{\circ} \mathrm{C}$ heat delivery.

- Platinized asbestos should be suitable for catalysing the dissociation reaction and vanadium pentoxide catalysts for the synthesis reaction are readily available.

- The system has little potential for energy storage through storage of the reaction products, made difficult because of the need to store low pressure oxygen. Nemecek et al. [8] have proposed that the thermochemical energy transport system be used in combination with eutectic salt storage of thermal energy, at the expense that heat delivery from the salt bath would be reduced to typically $350^{\circ} \mathrm{C}$ and the system efficiency reduced to $17 \%$.

4.3 Ammonia. - No thermodynamically stable side products are formed.

- High strength nickel alloys are used for cons- truction of the solar absorber. It has been shown elsewhere [21] that a long life thermochemical absorber could be mass produced for $\$ 10$ per square metre of collector area (1979 US dollars).

- Separation of the liquid reaction and gaseous product phases allows insolation variations at the absorber to be accommodated without the need for variation of the absorber feed [11].

- Promoted iron catalysts are available for the synthesis reaction. Dissociation catalysts (nickel on alumina) are presently available for low pressure operation. There is a need for further development of high activity ammonia dissociation catalysts suitable for the high pressure operation and conditions of thermal cycling [21].

- The proposed pressures are within the limits of current widespread industrial experience. High pressure operation leads to good heat transfer, but also to the necessity for expensive thick-walled vessels.

- Although ammonia is pungent and toxic, it is easily dispersed. All-welded construction leads to low losses (less than $0.1 \%$ ) in modern ammonia process plant.

- There is potential for long term lossless storage of energy through underground storage of the hydrogen-nitrogen gas mixture [23]. Ammonia vapour would need to be removed from the gas stream before storage.

4.4 Methanol. - This system is similar to the high pressure ammonia system except that the heat delivery is at lower temperatures and there would be considerable problems with side-product buildup in a closed system. Additional plant could be incorporated for reverting the side-products to primary compounds such as hydrogen and carbon monoxide, but only at extra expense. The low pressure methanol synthesis process developed by ICI [20] which is in widespread use today would be less suitable than the earlier high pressure process because of the lower quality of the recovered heat.

5. Summary and conclusions. - In this paper we have presented an outline of the thermodynamic and chemical engineering aspects of reversible chemical reactions that have been proposed for solar thermochemical power generation. It has been beyond the scope of the paper to present a fully detailed cost optimization as no prototype system has yet been tested and comparative figures are not available. Nevertheless, sufficient information exists to serve as the basis for comparison, to the extent that a satisfactory guide as to the optimum choice of system may be obtained. Particular emphasis has been placed on identifying all sources of thermodynamic irreversibility and thermal loss in order to highlight those areas of each system which need special attention and which need to be considered when new reaction systems are proposed in the future.

On the basis of the information presented in this 
paper, it is considered that of the four candidate systems for solar thermochemical power generation, that based on ammonia has the best potential for implementation in a first generation solar thermochemical power plant. This opinion is based on the satisfactory system efficiency and on the relatively straightforward chemical engineering design requirements for closed loop solar thermochemical operation. There is also potential for long term lossless energy storage through underground storage of the high pressure hydrogen-nitrogen mixture. Further development work is required in order to test the ammonia-based thermochemical energy transfer system on a pilot scale and also to verify the economic and technical viability of the solar thermochemical absorbers.

The sulphur trioxide system is characterised by the highest value of system efficiency but there are several problems which must be investigated at this stage if it is to be shown to be competitive. Further work is required to prove the economic and technical viability of the ceramic focal absorbers and to investigate the cost implications of the necessity to maintain hot pipelines in order to avoid sulphur trioxide solidification. There is also little potential for long term lossless storage of energy.
Both the water-methane and methanol systems have been shown to be characterised by comparatively low system efficiencies for solar thermochemical power generation, the former because of rejection of medium grade condensation heat which is difficult to utilize, and the latter because of low heat delivery temperature. The water-methane system is suitable, however, in a system in which the medium grade heat can be utilized as in the industrial heat distribution application for which it is primarily being developed.

It is hoped that further work in solar thermochemical power generation will be encouraged in the light of the satisfactory system efficiencies and the level of chemical engineering difficulty which is not beyond that consistent with modern chemical process plant expertise. Further encouragement should also ensue from the potential for solution of the energy storage problem that besets any system proposed for solar electricity generation.

Acknowledgment. - The author wishes to thank Dr P. O. Carden for the many informative conversations which have assisted in the development of the subject of this paper.

\section{References}

[1] Wentworth, W. E. and Chen, E., Sol. Energy 18 (1976) 205-214.

[2] Hilberath, E. and Teggers, H., West German Patent 1 (1968) 298, 233.

[3] Hafele, W., Science 84 (1974) 360-367.

[4] Kugeler, K., Nucl. Eng. Des. 34 (1) (1975) 65.

[5] Vakil, H. B. and Flock, J. W., Closed Loop Chemical Systems for Energy Storage and Transmission, General Electric Company (1978), ERDA Contract EY-76-C02-2676.

[6] Chubb, T. A., Sol. Energy 17 (1975) 124-136.

[7] Chubb, T. A., Nemecek, J. J. and Simmons, D. E., Sol. Energy 20 (1978) 219-224.

[8] NemeCeK, J. J., Simmons, D. E. and Chubb, T. A., Sol. Energy 20 (1978) 213-217.

[9] Carden, P. O., Energy Conversion Technical Report No 8 (1974), Department of Engineering Physics, The Australian National University, Canberra, Australia.

[10] Carden, P. O., Sol. Energy 19 (1977) 365-378.

[11] Williams, O. M. and CARDEN, P. O., Int. J. Energy Research 3 (1979) 129-142.

[12] Williams, O. M. and Carden, P. O., Int. J. Energy Research 3 (1979) 29-40.
[13] Carden, P. O. and Williams, O. M., Int. J. Energy Research 2 (1978) 389-406.

[14] HildebrandT, A. F., Solar Tower Thermochemical Energy Cycles, Hydrogen Energy Fundamentals SymposiumCourse, Miami, Florida (1975).

[15] Prengle, H. W. and Sun, C., Sol. Energy 18 (1976) 561-567.

[16] Williams, O. M. and Carden, P. O., Sol. Energy 22 (1979) 191-193.

[17] ICI Catalyst Handbook (1970), Wolfe, London.

[18] Stull, D. R. and PROPHET, H., JANAF Thermochemical Tables, 2nd edition (1971), NSRDS-NBS37, US Government Printing Office, Washington, DC.

[19] Williams, O. M., Energy Conversion Technical Report No 16 (1978), Department of Engineering Physics, The Australian National University, Canberra, Australia.

[20] Strelzoff, S., Chem. Eng. Prog. Symp. Ser. 66 (1970) 54-68.

[21] Williams, O. M., Design and Cost Analysis for an Ammonia Based Solar Thermochemical Cavity Absorber (1980), Sol. Energy, to be published.

[22] Williams, O. M., Sol. Energy 20 (1978) 333-342.

[23] Carden, P. O. and Paterson, L., Hydrogen Energy (1980), to be published. 